\title{
Optical nanosensing in cells
}

\section{Francesco Baldini}

Published online: 13 June 2013

C Springer-Verlag Berlin Heidelberg 2013

The advent of optical nanosensors for quantitative determination of bioanalytes at the intracellular level is one of the most fascinating new developments in nanoparticle and nanomaterial technology. Fluorescent labels have been used in microscopy for many years, as labels to locate bioanalytes at the cellular level and to follow biological pathways. In recent years, however, the possibility of developing optical nanosensors capable of obtaining quantitative information in real time about intracellular events, for example metabolite dynamics, activated gene expression, or efficiency of administered drugs, is an unprecedented development. For example, the "nanodevices" oligonucleotide optical switches can work as nanosensor and drug at the same time, by addressing specific RNA messengers and preventing overexpression of proteins associated with pathologic conditions; in this case nanosensing and drug activity are combined and the optical signal is associated with the efficiency of the drug. It should be said that, in general, to direct the nanosensors toward welldefined cellular targets, highly complex nanostructures must be developed. The overall strategy for detection of intracellular bioanalytes, delivery of optical nanosensors inside the cell, is not trivial but relies on conjugation of nanosized structures with different molecules capable of:

1. directing the nanostructures to the unhealthy cells;

2. binding to marker molecules differently expressed by these cells or interacting with chemicals of interest;

3. improving cell membrane penetration; and

4. providing optical signals related to the amount of the analyte investigated.

Published in the topical collection Optical Nanosensing in Cells with guest editor Francesco Baldini.

\section{F. Baldini $(\triangle)$}

IFAC-CNR,

Via Madonna del Piano 10,

50019 Sesto Fiorentino, Firenze, Italy

e-mail: baldini@ifac.cnr.it
Each of these steps requires particular attention and implies the use of well-defined procedures which have not still been established. A critical issue is associated with precise positioning of the nanosensors inside the cell, including directing them to the correct cellular compartment (cytosol, nucleus, organelles, etc.). In terms of the optical signal, fluorescence is surely the main optical transduction associated with intracellular sensors, because both measurement intensity and lifetime change as a function of the analyte investigated, with lifetime measurement having the undoubted advantage of eliminating, or at least largely attenuating, the autofluorescence coming from the cell environment. Plasmonic nanoprobes have recently been proposed as nanostructures capable of either providing strong surfaceenhanced Raman scattering or enhanced photoluminescence by means of plasmon resonance. Use of optical nanosensors in a real scenario is still far from being achieved - although many proofs of concept have been established and described in the literature, there are few examples of real quantitative determination of analytes at intracellular levels.

Another fascinating field of optical nanosensors is related to genetic modification of cells by introduction of bioluminescent reporters which are integrated within the cell. With this approach the whole cell acts as the optical sensing element and an optical signal is provided when the cell comes into contact with a specific analyte. Bacteria, yeasts, or mammalian cells are usually used for this purpose and this approach is, surely, very promising from the perspective of a novel generation of biosensors for environmental, medical, and food analysis.

All these aspects are included in the articles published in this issue on optical nanosensors, with, in particular, critical reviews on plasmonic nanoprobes and oligonucleotide optical switches, a trends paper on whole-cell bioluminescencebased sensors, and a forefront paper on novel cellpenetrating peptides combined with the use of quantum dots as intracellular transporting nanoparticles. 


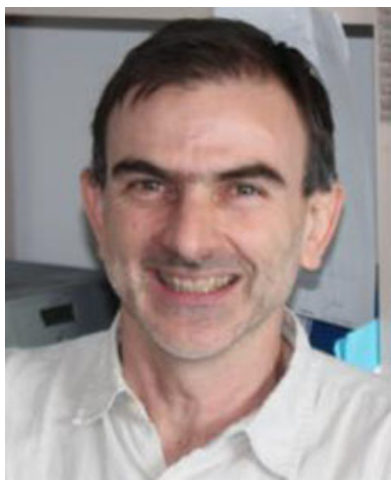

Francesco Baldini has been senior scientist at the Institute of Applied Physics of the National Council of Research, Italy, since 2001. His research activity has been devoted to the development of optical sensors and to the application of optical methods for restoration of paintings and frescoes. Now his research interests lie in optical fibre sensors and systems for chemical and biochemical applications. Since 2005 he has been member of the International Advisory Board of the journal Analytical and Bioanalytical Chemistry. In 2009 he was nominated fellow of SPIE for his achievements in biological and chemical sensing in biomedicine, and in January 2013 was elected vice-president of the Italian Society of Optics and Photonics. 